\title{
Drugs with anti-oxidant properties can interfere with cell viability measurements by assays that rely on the reducing property of viable cells
}

\author{
Niraj Shenoy, Mary Stenson, Joshua Lawson, Jithma Abeykoon, Mrinal Patnaik, Xiaosheng Wu and Thomas Witzig
}

Cell viability assays such as Cell Titer Blue and Alamar Blue rely on the reducing property of viable cells to reduce the reagent dye to a product which gives a fluorescent signal. The current manufacture-recommended protocols do not take into account the possibility of the reagent substrate being reduced directly to the fluorescent product by drugs with an anti-oxidant property. After suspecting spurious results while determining the cytotoxic potential of a drug of interest (DOI) with known anti-oxidant property against a renal cell cancer (RCC) cell line, we aimed to establish that drugs with anti-oxidant property can indeed cause false-negative results with the current protocols of these assays by direct reduction of the reagent substrate. We also aimed to counter the same with a simple modification added to the protocol. Through our experiments, we conclusively demonstrate that drugs with anti-oxidant properties can indeed interfere with cell viability measurements by assays that rely on the reducing property of viable cells. A simple modification in the protocol, as elaborated in the manuscript, can prevent spurious results with these otherwise convenient assays.

Laboratory Investigation (2017) 97, 494-497; doi:10.1038/labinvest.2017.18; published online 27 February 2017

\section{INTRODUCTION}

Cell viability assays such as Cell Titer Blue and Alamar Blue rely on the reducing property of viable cells and are commonly used as a screening method to determine the cytotoxic potential of drugs against cancer cells. The results obtained from these assays often determine whether or not the drugs are investigated further for their anti-cancer potential. The Cell Titer Blue and Alamar Blue assays use the indicator dye Resazurin to indicate cell viability by measuring the metabolic capacity of cells. Viable cells retain the ability to reduce Resazurin into Resorufin, which gives a fluorescent signal. https://www.promega.com/-/media/files/ resources/protocols/technical-bulletins/101/celltiter-bluecellviability-assay-protocol.pdf.

We aimed to determine the cytotoxic effect of a known anti-oxidant drug on the viability of RCC line RCC4, both individually at $5 \mathrm{mM}$ concentration and in combination with Azacytidine (Aza) $5 \mu \mathrm{M}$ after a $24 \mathrm{~h}$ exposure. The assay reagent was added directly to the media containing the drug, as stated in the protocol. To our surprise, the drug treatment wells had a substantially higher fluorescent signal (inferring increased viability) after the addition of Cell Titer Blue (incubation time $4 \mathrm{~h}$ ) compared to the control wells. The experiment was repeated, with the same result. However, on light microscopy examination of treated and control cells, the opposite seemed true. We therefore suspected that the test drug's anti-oxidant property may have reduced the Resazurin directly to the fluorescent Resorufin resulting in the increased fluorescent signal, providing a false-negative result, contrary to the assumption in the protocol that the reagent dye is only reduced intracellularly; and sought to establish the same. We also aimed to determine a solution for the interference, given the widespread use of these convenient assays in cancer research to determine the cytotoxic potential of drugs.

\section{MATERIALS AND METHODS}

The Cell Titer Blue assay was performed with the same drug concentration and exposure time, but this time with suctioning of the media containing the drug and washing the cells followed by incubation with fresh media and Cell Titer Blue reagent for $4 \mathrm{~h}$, before measuring the fluorescent signal with a plate reader. The rationale was to remove any remaining drug before the addition of the assay reagent, to avoid the direct reduction of Resozurin by the drug. This resulted in significantly reduced fluorescent signal in the treatment wells compared to the control wells indicating that

Division of Hematology, Mayo Clinic, Rochester, MN, USA

Correspondence: Dr N Shenoy, MD or T Witzig, MD, Division of Hematology, Mayo Clinic, 200 First Street SW, Rochester 55905, MN, USA.

E-mail: shenoy.niraj@mayo.edu or witzig.thomas@mayo.edu

Received 30 November 2016; revised 26 December 2016; accepted 28 December 2016 
a

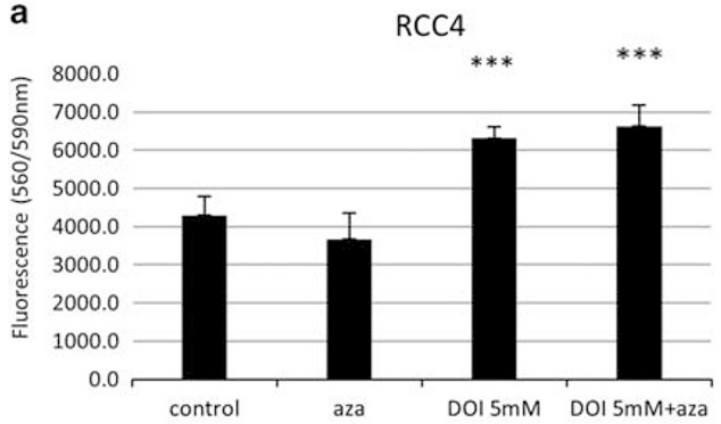

C

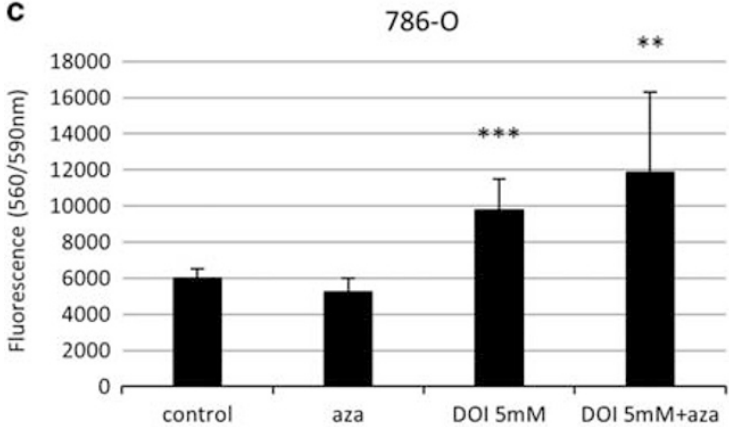

b

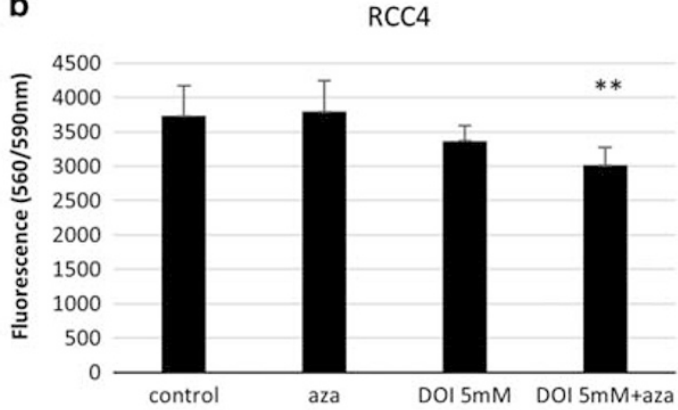

d

786-0

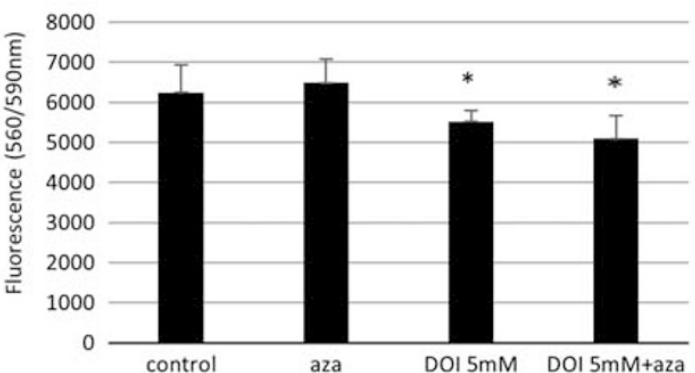

Figure 1 (a) Cell viability assay of the RCC4 cell line treated with Azacytidine and the drug of interest (DOI) with known anti-oxidant properties with the Cell Titer Blue protocol. As per manufacturer's instructions the Cell Titer Blue reagent was added directly to the media with the treatment drugs. The results suggest that the DOI may be enhancing viability (two-tailed $t$-test ' $P$ ' values for DOI $5 \mathrm{mM}$ and DOI $5 \mathrm{mM}$ +aza in comparison to control7.7E- 06 and 1.6E-05, respectively). (b) Same protocol and cell line (RCC4) except removal of the media containing the drug and washing the cells prior to incubation with fresh media and Cell Titer Blue reagent. This reveals the drug to be reducing cell viability (' $P$ ' values for DOI $5 \mathrm{mM}$ and DOI $5 \mathrm{mM}+$ aza in comparison to control-0.1 and 0.004, respectively). (c, d) Same experiment as (a and $\mathbf{b}$ ) on a different RCC cell line 786-O. (c) ' $P$ ' values for DOI $5 \mathrm{mM}$ and DOI $5 \mathrm{mM}+$ aza in comparison to control—0.0004 and 0.009 , respectively. (d) ' $P$ ' values for DOI $5 \mathrm{mM}$ and DOI $5 \mathrm{mM}+$ aza in comparison to control-0.04 and 0.01 , respectively.

indeed the test drug did have the expected cytotoxicity. The same set of experiments (with both the manufacturerecommended protocol and the modified version) was performed with another RCC line 786-O with similar results (Figure 1).

The DOI was then tested on another cancer cell line (lymphoma-LY1) with the manufacture-recommended protocol to ensure that this phenomenon was not unique to certain types of cancer cell lines. The false-negative results were also seen with the lymphoma cell line (Figure 2).

Finally, in order to conclusively prove that the DOI directly reduced the reagent substrate Resazurin to the fluorescent Resorufin, the experiment was performed without cells. The Alamar Blue assay reagent (which also contains Resazurin) was added to fresh media with and without the DOI and incubated for $20 \mathrm{~min}$. The wells with the drug showed significantly increased fluorescence compared to the wells without the drug, conclusively proving that drugs with antioxidant properties can reduce the reagent substrate directly, even without cells (Figure 3).

\section{RESULTS AND DISCUSSION}

We have demonstrated that drugs with anti-oxidant properties can interfere with cell viability measurements when assays that rely on the reducing property of viable cells are used to assess their cytotoxic potential with the current manufacturerecommended protocol. This interference can however be prevented with a small modification of the protocol-to suction the culture media containing the drug and to wash the cells with phosphate buffered saline prior to incubation with fresh media and the assay reagent, especially when the redox property of the drug being tested is unknown or known to be high. Other viability assays that rely on the reducing property of viable cells such as MTT assay (which is a colorimetric assay that relies on the reduction of 3-(4,5-dimethylthiazol-2yl)-2,5diphenyltetrazolium bromide to its insoluble formazan, which has a purple color) would also likely benefit from the addition of this step. Given the widespread use of these assays and the lack of this step in the manufacturers' instructions, we believe that this observation is of significant importance to the cancer research community worldwide, to avoid spurious results while testing the cytotoxic potential of drugs with a high redox potential using these assays, which are otherwise very convenient methods of determining the effect of drugs on cell viability. How this seemingly obvious phenomenon escaped detection during the development of Resazurin-based cell viability assays and its theoretical impact on cancer research: the Resazurin reduction test has been used for more 

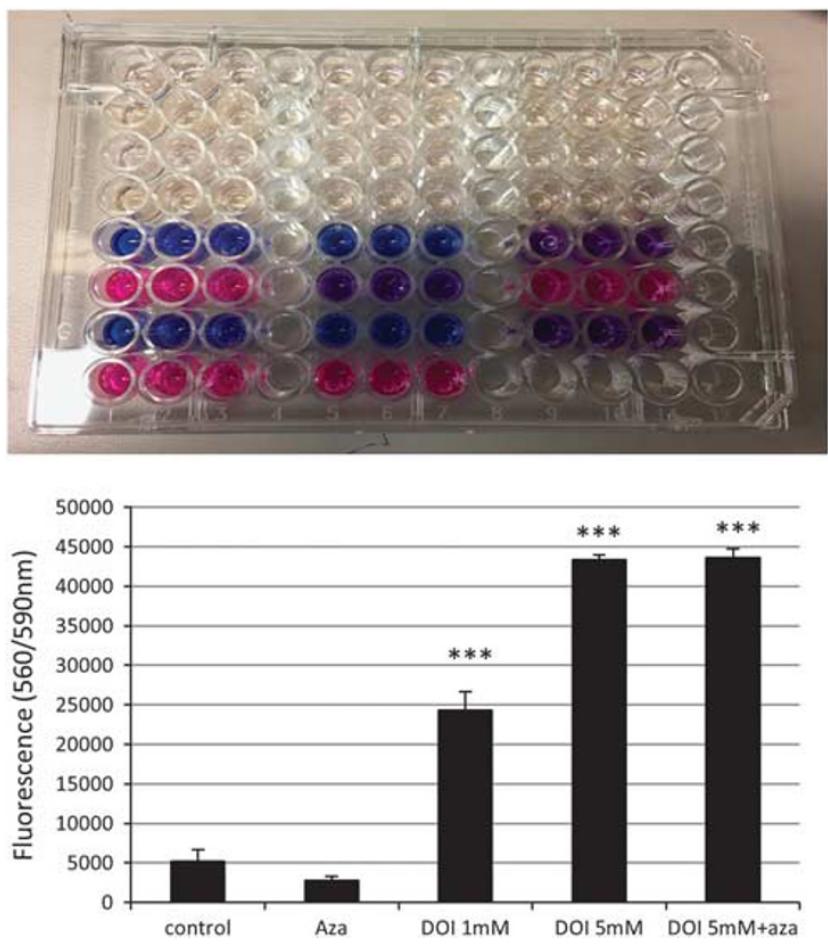

Figure 2 Representative picture of the Cell Titer Blue assay with a lymphoma cell line LY-1. The blue wells (nonfluorescent) are control or Azacytidine treatment wells, the purple wells (medium fluorescence) are with $1 \mathrm{mM}$ drug of interest, and the pink wells (highest fluorescence) are with $5 \mathrm{mM}$ drug of interest, without and with Azacytidine; falsely implying that the viability of the cells increased with increase in drug concentration. The bar graph represents the wells in the picture (twotailed $t$-test ' $P$ ' values for DOI $1 \mathrm{mM}$, DOI $5 \mathrm{mM}$, and DOI $5 \mathrm{mM}+$ aza in comparison to control-1.16E-08, 4.7E-14, and 2.27E-13, respectively).

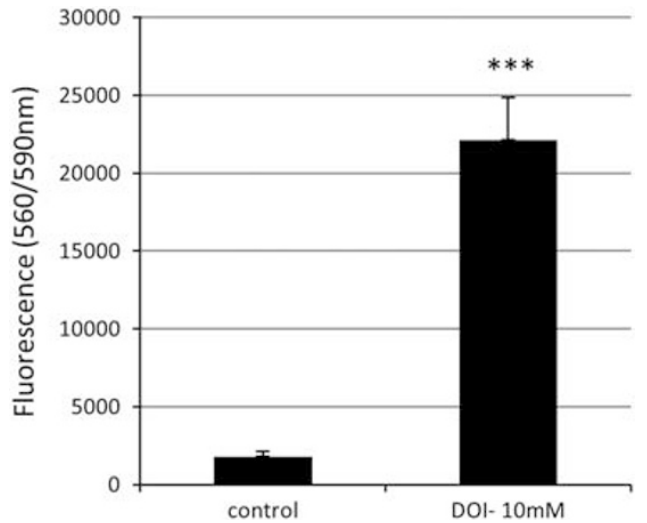

Figure 3 Alamar Blue assay reagent added directly to fresh media without cells; with and without the drug of interest (DOI). After incubation for $20 \mathrm{~min}$, wells with the DOI had substantially higher fluorescence signal, clearly demonstrating that drugs with anti-oxidant properties can directly reduce Resozurin to the fluorescent Resorufin, even in the absence of cells (two-tailed $t$-test ' $P$ ' value 0.0002 ).

than 60 years to detect bacterial and yeast contamination of milk, and to assess the quality of semen. In the 1990s, it began gaining popularity as a convenient method of assessing

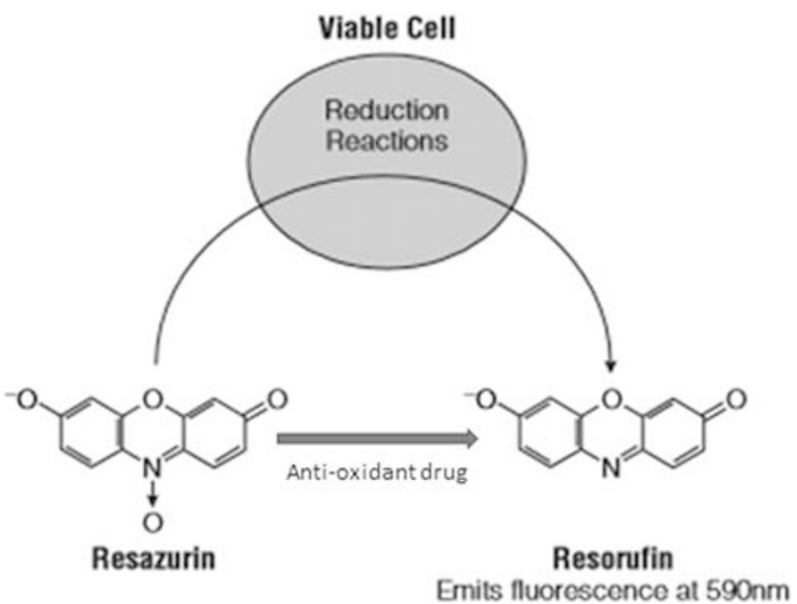

Figure 4 Picture depicting the interference of drugs with anti-oxidant properties on Cell Titer Blue and Alamar Blue assays by direct reduction of Resazurin to Resorufin.

cytotoxicity. However, before the year 2000, it was not clear whether the Resazurin dye was reduced within the cells or in the 'reduced medium' from increased cell growth. In the year 2000, O'Brien et al. reported that when the dye was added to the reduced medium separated from cells after a $24-48 \mathrm{~h}$ incubation, it did not get converted to the fluorescent Resorufin. They detected fluorescence in the cytoplasm of cells on addition of the dye, albeit at a quantity not large enough to be detected by the fluorescent microplate reader. They therefore concluded that the dye likely enters the cytoplasm where it is reduced to the fluorescent product, which is then excreted into the medium giving the fluorescent signal. ${ }^{1}$ Furthermore, an analysis of hepatic subcellular fractions suggested that Resazurin can be reduced by mitochondrial, cytosolic and microsomal enzymes. ${ }^{2}$ When this was extrapolated into cancer research to test cell viability, the question of how the redox property of drugs tested would affect the dynamics seems to have escaped widespread attention. It was assumed that the dye could only get reduced intracellularly. The Cell Titer Blue protocol even mentions 'cell washing, removal of medium and multiple pipetting steps are not required' and states the 'Test compound control' to be optional.

Most anti-cancer drugs developed, initially go through cell viability assays or proliferation assays. The decision to pursue further research with these agents is often based on their effects on cell viability or proliferation. One therefore wonders how many indigenous agents with high redox potential tested with assays that rely on the reducing property of viable cells had spurious results. The addition of a simple step, as elaborated above, can eliminate such spurious results from these otherwise convenient assays, and we recommend that this step be incorporated in the protocols of the above mentioned assays, especially when testing a drug with high 
redox potential, and to always have a 'test compound' control without cells (Figure 4).

\section{DISCLOSURE/CONFLICT OF INTEREST}

The authors declare no conflict of interest.
1. O'Brien J, Wilson I, Orton $\mathrm{T}$, et al. Investigation of the Alamar Blue (resazurin) fluorescent dye for the assessment of mammalian cell cytotoxicity. Eur J Biochem 2000;267:5421-5426.

2. Gonzalez RJ, Tarloff JB. Evaluation of hepatic subcellular fractions for Alamar Blue and MTT reductase activity. Toxicol In Vitro 2001;15: 257-259. 